\title{
Metabolismo energético en ovejas gestantes esquiladas y no esquiladas sometidas a dos planos nutricionales. Efecto sobre las reservas energéticas de sus corderos
}

\author{
Energy metabolism in shorn and unshorn pregnant ewes under two different nutritional planes. \\ Effects on the energetic storage of their lambs \\ L Cal-Pereyra ${ }^{a}$, A Benech ${ }^{\mathrm{b}}$, S Da Silva ${ }^{\mathrm{b}}$, A Martín ${ }^{\mathrm{a}}$, JR González-Montaña ${ }^{\mathrm{c}^{*}}$ \\ a Departamento de Patología, Universidad de la República, Montevideo, Uruguay. \\ b Departamento de Fisiología, Universidad de la República, Montevideo, Uruguay. \\ ${ }^{c}$ Departamento de Medicina, Cirugía y Anatomía Veterinaria, Facultad de Veterinaria, Universidad de León, León, España.
}

\begin{abstract}
SUMMARY
The aim of this work was to evaluate the role of nutrition and shearing during last third of pregnancy, on the energetic metabolism of ewes and the energetic storage of their lambs at parturition. 38 Corriedale ewes carrying single fetus were randomly divided in two groups at 90 days of pregnancy, $\mathrm{R}(\mathrm{n}=12$, supplemented with ruminant concentrate) and $\mathrm{CN}(\mathrm{n}=26$, fed on natural pastures). The energetic status of the ewes was evaluated using glicaemia and seric levels of ß-hidroxybutyrate. When reaching 110 days of gestation, half of the ewes in CN group were shorn (E). Newborn lambs were blooded to study glicaemia levels at parturition and $24 \mathrm{~h}$ afterwards, weighed at birth and after $24,48 \mathrm{and} 72 \mathrm{~h}$, and total weight and energetic value of their perirenal brown fat was measured. Ewes in groups R and E presented glicaemia levels higher than those in $\mathrm{CN}$ in the days before at parturition, whereas hydroxybutyrate was more elevated in groups $\mathrm{CN}$ and $\mathrm{E}$ than in group $\mathrm{R}$. The weight of ewe-lambs at birth did not differ between groups, but by this time glicaemia levels were higher in group R than in CN. Glicaemia was also higher in lambs of both groups R and E by $24 \mathrm{~h}$ after birth. Perirenal brown fat in group $\mathrm{E}$ had highter caloric value compared to $\mathrm{CN}$, which suggests a relationship between the energetic status and management of ewes at parturition and the energetic storage of their lambs at birth.
\end{abstract}

Palabras clave: nutrición materna, esquileo, cordero, reserva energética.

Key words: maternal nutrition, shearing, lambs, energetic storage.

\section{INTRODUCCIÓN}

La obtención de un elevado número de corderos destetados por oveja reproductora constituye el objetivo básico de la producción ovina. Son varias las razones por las cuales debe maximizarse este índice, ya que los corderos producidos serán los futuros reemplazos reproductivos, los elementos sobre los cuales se ejercerá presión de selección y el objetivo final en la producción de carne y lana.

En la mayoría de los países donde se produce el ganado ovino las pérdidas de corderos al destete se sitúan entre un 15 y un 20\% de los corderos nacidos (Dennis 1974, Corner y col 2005, Dwyer y Morgan 2006). Estas muertes se producen fundamentalmente durante o de inmediato después del parto, y principalmente en los primeros tres días de vida, citándose como principales causas la inanición y la exposición al frío (Azzarini 1974, Mari 1987).

Inmediatamente después del parto el cordero es sometido a la acción directa del medio ambiente, y debe poner en funcionamiento sus mecanismos de termorregulación. La

Aceptado: 04.08.2011.

* jramirogonzalez@unileon.es pérdida de calor puede ser debida a la evaporación de los líquidos fetales, la lluvia, la temperatura ambiente y a las corrientes de aire (Faurie y col 2004), siendo los corderos más livianos los que mayor cantidad de calor pierden, ya que tienen mayor relación área/peso corporal (Stephenson y col 2001).

La generación de calor se produce fundamentalmente por dos vías, una física dependiente de las contracciones musculares debidas a los escalofríos que son responsables de aproximadamente el 55\% del calor total producido y una segunda bioquímica, gracias a la combustión de la grasa parda, que proporciona el restante $45 \%$ del calor (Encinias y col 2004, Clarke y Symonds 1998). Este último proceso es muy importante en el ganado ovino, ya que al parto el $100 \%$ de sus reservas son de grasa parda (Encinias y col 2004). Este tipo de grasa, presente en los grandes mamíferos y especialmente en las dos últimas semanas de gestación, se localiza principalmente alrededor de ciertas vísceras como riñones y corazón (McFarlane 1965, Avram y col 2005). Posee una importante irrigación sanguínea y un alto valor oxidativo energético (Clarke y col 1997b). Durante la exposición al frío la irrigación de la grasa parda puede llegar a quintuplicarse, lo que demuestra la importancia fundamental de este tejido para la producción de calor en los corderos (Encinias y col 2004). 
La glucosa es un metabolito de enorme importancia en el metabolismo energético de la futura madre. Es el principal sustrato energético a nivel cerebral, es además fundamental para la síntesis de triglicéridos, la contracción muscular, la síntesis de lactosa en la glándula mamaria y para el aporte de energía al feto (Pell y Bergman 1983, Lindsay y Setchell 1976).

El desarrollo y el crecimiento de algunos tejidos fetales, altamente especializados, es más costoso, en términos de nutrientes y necesita más alimento por unidad de peso ganado que en el animal adulto (Gibbons 1996). El requerimiento energético para la unidad feto-placenta puede llegar a representar hasta el $45 \%$ de la glucosa materna y el $72 \%$ de la oferta de aminoácidos maternos (Bell 1995). El incremento de los requerimientos al final de la gestación está causado por el hecho de que cerca del $85 \%$ del crecimiento fetal ocurre durante las últimas seis semanas de la gestación (Rook 2000, Robinson 1996, Russel 1984). El aumento de las demandas fetales puede ser de tal magnitud que Rook (2000) sugiere que en la gestación avanzada los requerimientos energéticos de las ovejas aumentan sobre los de mantenimiento hasta un $150 \%$ en aquellas ovejas que gestan un solo feto y hasta un $200 \%$ en ovejas con gestaciones dobles. Por ello existe una estrecha relación entre el nivel de nutrición de la oveja durante este período y el peso del cordero al nacimiento (Osgerby y col 2002). Además el peso corporal de las ovejas al parto ejerce una influencia crítica sobre el peso de la placenta, el tamaño de los corderos al nacimiento y la supervivencia postnatal (Clarke y col $1997^{\mathrm{c}}$ ).

El esquileo realizado un mes antes del parto aumenta el consumo de alimento por las ovejas (Vipond y col 1987). Según Revell y col (2000) el incremento del peso en los corderos nacidos de ovejas esquiladas está asociado a un aumento del ingreso de la glucosa no insulinodependiente a la unidad fetoplacentaria. Se ha demostrado además que los corderos nacidos de ovejas esquiladas presentan mayor tamaño y mayor cantidad de grasa parda, con mayor actividad termogenética, que aquellos nacidos de ovejas no esquiladas; registrándose además un incremento en la irrigación de la grasa parda como consecuencia del estrés producido por la disminución de la temperatura corporal de las ovejas (Clarke y col 1997a , Gate y col 1999, Encinias y col 2004). El estrés por frío, inducido por el esquileo, parece inhibir la secreción de insulina dando lugar a un aumento de la glucemia (Symonds y col 1986, Symonds y col 1988). El incremento de la glucosa circulante es directamente aprovechado por la glándula mamaria para la producción de lactosa, lo que incrementa el valor nutricional del calostro producido $\mathrm{y}$, secundariamente, contribuye a hacerlo menos viscoso (Banchero y col 2004 ${ }^{\mathrm{b}}$, Banchero y col 2006).

La concentración de cuerpos cetónicos en sangre es un buen indicador del estado energético de las ovejas en la gestación y permiten conocer si sus requerimientos energéticos están siendo satisfechos (Russel 1984, Rhind
2004, McMullen y col 2005). Russel y col (1977) estimaron, en función de los valores del B-Hidroxibutirato (BHOB), el grado de nutrición de ovinos en condiciones extensivas y establecieron las siguientes categorías: nutrición normal $=\mathrm{BHOB}<0,71 \mathrm{mmol} / \mathrm{l}$, subnutrición modera$\mathrm{da}=\mathrm{BHOB}<1,1 \mathrm{mmol} / \mathrm{l}$ y subnutrición severa $=\mathrm{BHOB}$ $>1,6 \mathrm{mmol} / 1$. Andrews (1997) propuso una escala similar para evaluar la condición nutricional de ovejas gestantes: nutrición normal $=\mathrm{BHOB}<0,8 \mathrm{mmol} / \mathrm{l}$, moderada subnutrición = valores de $\mathrm{BHOB}$ entre 0,8 y 1,6 mmol/l y valores de $\mathrm{BHOB}>1,6 \mathrm{mmol} / 1$ indican severa subnutrición.

En el presente trabajo se pretende evaluar si los corderos nacidos de ovejas que han recibido un suplemento nutricional en el último tercio de la gestación, o bien de aquellas que han sido esquiladas en el día 110 de preñez, poseen mayor peso y mayores reservas energéticas al nacimiento que los nacidos de ovejas control.

\section{MATERIAL Y MÉTODOS}

Se utilizaron animales alojados en el Campo Experimental $\mathrm{N}^{\mathrm{o}} 2$ (Libertad) de la Facultad de Veterinaria de la Universidad de la República, Uruguay, situado en el Departamento de San José ( $\left.34^{\circ} 38^{\prime} \mathrm{S} ; 56^{\circ} 39^{\prime} \mathrm{W}\right)$, entre los meses de febrero y agosto de 2006. El protocolo experimental fue aprobado por la Comisión Honoraria de Experimentación Animal (CHEA) de dicha Universidad.

Las temperaturas medias oscilaron en este periodo entre $25,3^{\circ}$ y $5,3{ }^{\circ} \mathrm{C}$, con temperaturas máxima de $32{ }^{\circ} \mathrm{C}$ a mediados de febrero y mínima de $0,5^{\circ} \mathrm{C}$ en los primeros días de agosto. La humedad relativa osciló entre el 50 y $100 \%$, y si bien la mayoría de los días no llovió, en la última semana de febrero se registraron $125 \mathrm{~mm}$ de agua/día.

\section{DISEÑO EXPERIMENTAL}

Se sincronizaron los celos de 71 ovejas Corriedale adultas (4 a 6 años), identificadas por medio de crotales numerados, mediante esponjas intravaginales que contenían $60 \mathrm{mg}$ de medroxiprogesterona (Sincrovin $®$, Santa Elena) durante 12 días. Una vez retiradas las esponjas, las ovejas fueron cubiertas, mediante monta natural, por dos carneros de la misma raza con arneses marcadores. El control de las montas se realizó durante cuatro días, registrándose el día de la cubrición como día cero $(0)$ de la gestación. A los 60 días se realizó el diagnóstico de gestación mediante ultrasonografia (Buckrell 1988), descartándose las ovejas vacías y aquellas con gestaciones múltiples. Así fueron seleccionadas 38 ovejas gestando un solo feto.

Posteriormente a la cubrición, todos los animales pasaron a alimentarse en una pradera con pasto natural compuesta fundamentalmente por Cynodon dactylon. Cada $100 \mathrm{~g}$ de pasto (MS) proporcionaron 8,72\% de proteína bruta y 1,52 Mcal/kg MS de energía metabolizable (Laboratorio de Nutrición, Facultad de Veterinaria, Universidad de la República, Uruguay). Esta pradera se reservó para el 
presente trabajo experimental, de modo que ningún animal se alimentó en ella hasta el ingreso de nuestras ovejas.

A partir del día 90 las ovejas se dividieron en dos grupos. Un grupo estuvo compuesto por 12 animales denominado grupo ración $(\mathrm{R})$ que se alimentó en $1 / 3$ de la pradera y suplementando su alimentación con una ración balanceada (ración González Lamela para lanares, $n^{\circ}$ 2, cuadro 1). Se administraron $4.800 \mathrm{~g}$ de concentrado para todo el grupo, lo que aproximadamente permitió ingerir unos 400 gramos/día a cada oveja, distribuido en dos comidas. Otro grupo, denominado grupo campo natural $(\mathrm{CN})(\mathrm{n}=26)$, se alimentó con los $2 / 3$ restantes de la pradera y únicamente con pasto.

En el día 110 de gestación se esquiló (esquila preparto) a la mitad de las ovejas del grupo Campo Natural. Por tanto la totalidad de las ovejas quedan distribuidas en tres grupos: grupo ración $(\mathrm{R})(\mathrm{n}=12)$, grupo esquileo $(\mathrm{E})(\mathrm{n}=13) \mathrm{y}$ grupo campo natural $(\mathrm{CN})(\mathrm{n}=13)$, que fue considerado como grupo control. El aporte de pasto fue similar para los tres grupos, ya que se trató de la misma pradera (con idéntica composición botánica) y que fue subdividido en partes iguales mediante un alambrado eléctrico. La superficie del recinto donde se alojaron entre el día 90 y 140 de la experiencia fue de 8 ha, lo que permitió alimentarse sin problemas a la totalidad de los animales.

Todos los partos se produjeron entre los días $143 \mathrm{y}$ 153 de gestación, controlándose los mismos durante las 24 horas del día.

\section{PESO VIVO DE LAS OVEJAS}

Las ovejas fueron pesadas a las 9:00 horas de la mañana, mediante una balanza digital para ovinos, con una sensibilidad de $0,1 \mathrm{~kg}$. Las mediciones se hicieron a los 90 días de gestación, coincidiendo con el inicio de la alimentación diferenciada, en el día 110, coincidiendo con el esquileo y en el día 140 de gestación y en ningún caso se dejaron a las ovejas en ayunas para hacer el pesaje.

\section{DETERMINACIONES EN SANGRE DE LAS OVEJAS}

Las ovejas se muestrearon a los 90, 110 y 140 días de gestación. Las muestras de sangre, obtenidas por punción de la vena yugular, se utilizaron para la determinación de glucemia y $ß$-hidroxibutirato (BHOB). Se utilizaron tubos al vacío y agujas $18 \mathrm{G}$. La glucemia se midió a partir de sangre recogida en tubos con fluoruro de sodio y EDTA, antes de 12 horas tras el muestreo, utilizando un método enzimático colorimétrico (Glucose Liquicolor ${ }$, Human) y efectuando la lectura en un fotocolorímetro digital (Humalyser Junior) a una $\lambda=500 \mathrm{~nm}$. El BHOB se midió, una vez extraído el suero, con el kit comercial Ranbut ${ }^{\circledR}$ (Randox Laboratories Ltd.) en el fotocolorímetro anteriormente indicado y a una $\lambda=330 \mathrm{~nm}$.

\section{DETERMINACIONES EN SANGRE DE LOS CORDEROS}

Inmediatamente tras el nacimiento y 24 horas después se obtuvieron muestras sanguíneas de todos los corderos mediante punción de la vena yugular. Se utilizaron tubos al vacío y agujas $21 \mathrm{G}$. Se determinó la glucemia de manera similar a lo indicado para las ovejas.

\section{PESO VIVO DE LOS CORDEROS}

Se registraron los pesos vivos de todos los corderos inmediatamente tras el nacimiento y a las 24,48 y 72 horas de vida. Se utilizó una balanza mecánica con una sensibilidad de $0,01 \mathrm{~kg}$.

La ganancia relativa de peso de los corderos fue calculada mediante la siguiente fórmula:

$\frac{\mathrm{kg} \text { peso vivo a las } 72 \mathrm{~h}-\mathrm{kg} \text { peso vivo al nacimiento }}{\mathrm{kg} \text { peso viso a las } 72 \mathrm{~h}} \times 100$

\section{NECROPSIAS DE LOS CORDEROS}

Inmediatamente tras el parto cinco corderos de cada grupo (R, E y CN) fueron sacrificados mediante la aplicación intravenosa de una sobredosis de tiopental sódico (Tiopental sódico®, Laboratorio Cruz del Sur). Durante las necropsias se disecó totalmente la grasa parda perirrenal, la cual se pesó y posteriormente se extrajo una muestra para medir su valor energético expresado en calorías por gramo de materia seca (cal/g MS). Se mantuvo congelada a $-20{ }^{\circ} \mathrm{C}$ hasta su procesamiento en una bomba calorimétrica.

\section{PRODUCCIÓN DE CALOSTRO}

Se recogió el calostro de las ovejas a las que se les sacrificaron sus corderos dentro de la primera hora postparto y 24 horas después del parto. Tres minutos después de la administración i.m. de 10 UI de oxitocina (Hipofamina®, Dispert.) se consiguió el vaciado completo de la ubre mediante ordeño manual (O'Doherty y Crosby 1997). El calostro se midió mediante un matraz aforado.

\section{VALORACIÓN DEL PASTO Y DEL CONCENTRADO}

Se colectaron muestras del pasto y del concentrado utilizado en la alimentación de los animales. Se evaluaron la materia seca (MS), cenizas (Cen), fibra neutro detergente (FND), fibra ácido detergente (FAD), proteína bruta (PB) y energía metabolizable por kg de materia seca (EM/kg MS).

\section{ANÁLISIS ESTADÍSTICO}

Los análisis estadísticos se realizaron con el programa Statistica 6.0 (Stat Soft Inc, 1995). Todos los valores 
se presentan como valor medio \pm desviación estándar $(\bar{\chi} \pm \mathrm{DE})$. Tras comprobar la distribución normal de los datos encontrados, las diferencias entre los distintos grupos para la glucemia de las madres y de los corderos, el BHOB, los pesos vivos de las madres y de sus corderos, el volumen de calostro al parto y el producido a las 24 horas y el peso y la energía de la grasa parda de los corderos se evaluaron mediante un análisis de varianza (ANOVA), seguido del test de Tukey. Se consideraron diferencias significativas cuando $\alpha<0,05$.

\section{RESULTADOS}

Los resultados del análisis del concentrado, así como del pasto con los que fueron alimentadas las ovejas durante la experiencia se muestran en el cuadro 1.

En el día 90 de gestación no hubo diferencias estadísticamente significativas entre el peso vivo de las ovejas de los tres grupos ( $\mathrm{R}, \mathrm{E}$ y $\mathrm{CN})$. Los valores medios fueron $52,39 \pm 5,19 \mathrm{~kg}, 52,93 \pm 4,68 \mathrm{~kg}$ y $52,27 \pm 4,51 \mathrm{~kg}$ para los grupos R, E y CN, respectivamente. Tampoco se encontraron diferencias estadísticamente significativas en el día del esquileo (día 110 de gestación), siendo los pesos medios para los grupos $\mathrm{R}, \mathrm{E}$ y $\mathrm{CN}$ de $53,95 \pm 5,04 \mathrm{~kg}$, $51,86 \pm 4,45 \mathrm{~kg}$ y de $50,83 \pm 4,02 \mathrm{~kg}$, respectivamente. Sin embargo, en el día 140 de gestación las ovejas pertenecientes a los grupos $\mathrm{R}(57,29 \pm 4,86 \mathrm{~kg})$ y $\mathrm{E}(56,01 \pm 4,33 \mathrm{~kg})$

Cuadro 1. Análisis de composición del concentrado usado como suplemento y del pasto.

Analysis of concentrate and pasture composition.

\begin{tabular}{lcr}
\hline Componentes & Concentrado & Pasto \\
\hline Materia Seca (MS) & 89,5 & 42,36 \\
Cenizas (Cen) & 5,1 & 9,35 \\
Proteína Bruta (PB) & 12,5 & 8,72 \\
Fibra Neutro Detergente (FND) & & 76,22 \\
Fibra Acido Detergente (FAD) & 10,1 & 49,61 \\
Energía Metabolizable (EM) & 2,6 & 1,52 \\
\hline
\end{tabular}

(EM se presenta en Mcal/kg de MS y fue estimada en base al NRC). alcanzaron un peso significativamente mayor $(\mathrm{P}<0,005 \mathrm{y}$ $\mathrm{P}<0,01)$ que aquellas que se alimentaron exclusivamente con pasto natural $(52,04 \pm 4,14 \mathrm{~kg})$. Aunque el peso medio de las ovejas suplementadas con concentrado fue más alto que las esquiladas, esta diferencia no mostró carácter significativo.

Tampoco la glucemia medida en el día 90 de gestación mostró diferencias estadísticas entre los tres grupos (cuadro 2). En el día del esquileo la glucemia de las ovejas del grupo R mostró valores superiores que la de las madres de los demás grupos, aunque sin significación estadística. Como puede observarse en el cuadro 2, en el día 140 de preñez la glucemia de los tres grupos disminuyó de forma considerable, con valores incluso inferiores a los encontrados en el día 90 de gestación. En este momento la glucemia del grupo $\mathrm{R}$ fue significativamente mayor ( $\mathrm{P}<0,0001$ y $\mathrm{P}<0,037)$ al compararla con la de los grupos $\mathrm{CN}$ y E respectivamente. Asimismo la glucemia de las madres del grupo $\mathrm{E}$ fue significativamente más alto $(\mathrm{P}<0,001)$ que las del grupo $\mathrm{CN}$ (cuadro 2).

Los valores de BHOB sérico medido en el día 90 y en el día 110 de gestación no mostraron diferencias significativas entre los tres grupos. Los valores de este cuerpo cetónico en sangre, en el día 140 de gestación, fueron significativamente más altos $(\mathrm{P}<0,005)$ en las ovejas alimentadas exclusivamente a campo natural que en las del grupo R. También en el mismo día las ovejas del grupo $\mathrm{E}$ presentaron valores superiores $(\mathrm{P}<0,05)$ que las del grupo $\mathrm{R}$, aunque estas diferencias no fueron significativas (cuadro 2).

Aunque sin carácter significativo, el volumen de calostro obtenido tras el parto fue mayor en las ovejas suplementadas con concentrado (R), mientras que el más bajo se obtuvo de las madres del grupo CN (cuadro 3). A las 24 horas del parto el calostro producido se incrementó en más del doble en los tres grupos, manteniéndose las diferencias de forma similar a lo encontrado en el primer registro. Tampoco mostró significación estadística entre los grupos (cuadro 3). La producción de calostro presentó, tanto en el primero como en el segundo ordeño, una variación muy amplia en los tres grupos. Así para el primer ordeño, en los grupos R y E osciló desde 120 a 320 ml, mientras que en el grupo CN varió desde 50 a $290 \mathrm{ml}$. A las 24 horas la variación individual fue de 450 a $600 \mathrm{ml}$

Cuadro 2. Niveles maternos de glucemia y del $\beta$-hidroxibutirato de los diferentes grupos. Maternal levels of glicaemia and $\beta$-hydroxybutyrate.

\begin{tabular}{|c|c|c|c|c|c|c|}
\hline & \multicolumn{2}{|c|}{ Grupo Ración (R) } & \multicolumn{2}{|c|}{ Grupo Esquileo (E) } & \multicolumn{2}{|c|}{ Grupo Campo Natural(CN) } \\
\hline & Glicemia & $\mathrm{BOHB}$ & Glicemia & $\mathrm{BOHB}$ & Glicemia & BOHB \\
\hline Día 90 de gestación & $68,85 \pm 12,02$ & $0,36 \pm 0,13$ & $68,24 \pm 13,67$ & $0,36 \pm 0,11$ & $69,00 \pm 7,47$ & $0,30 \pm 0,12$ \\
\hline Día 110 de gestación & $74,25 \pm 10,79$ & $0,34 \pm 0,14$ & $70,18 \pm 15,45$ & $0,34 \pm 0,17$ & $70,49 \pm 14,36$ & $0,31 \pm 0,11$ \\
\hline Día 140 de gestación & $56,65 \pm 7,12^{\mathrm{a}}$ & $0,47 \pm 0,21^{\mathrm{d}}$ & $49,72 \pm 5,58^{b}$ & $0,69 \pm 0,25$ & $41,26 \pm 6,01^{\mathrm{c}}$ & $0,91 \pm 0,41^{\mathrm{e}}$ \\
\hline
\end{tabular}

Medias \pm DE. Glucemia (mg/dl), $\beta$-hidroxibutirato $(\beta-H O B, m m o l / l)$. Diferencias estadísticas: ${ }^{\mathrm{a}-\mathrm{b}} \mathrm{P}<0,037 ;{ }^{\mathrm{a}-\mathrm{c}} \mathrm{P}<0,0001 ;{ }^{\mathrm{b}-\mathrm{c}} \mathrm{P}<0,001 \mathrm{y}^{\mathrm{d}-\mathrm{e}} \mathrm{P}<0,005$. 
Cuadro 3. Volumen de calostro (mL) de los diferentes grupos.

Colostrum volume $(\mathrm{mL})$ of different groups.

\begin{tabular}{lccc}
\hline & Grupo Ración (R) & Grupo Esquileo (E) & Grupo Campo Natural (CN) \\
\hline Parto & $222 \pm 76,6$ & $200 \pm 74,8$ & $146 \pm 91,0$ \\
24 horas & $516 \pm 56,8$ & $506 \pm 176,3$ & $378 \pm 165,1$ \\
\hline
\end{tabular}

(Medias $\pm \mathrm{DE}$ ).

Cuadro 4. Peso vivo de los corderos (g) de los diferentes grupos. Lamb live weight ( $\mathrm{g}$ ) of different groups.

\begin{tabular}{lccc}
\hline & Grupo Ración $(\mathrm{R})$ & Grupo Esquileo $(\mathrm{E})$ & Grupo Campo Natural $(\mathrm{CN})$ \\
\hline Parto & $4441 \pm 421,9$ & $4419 \pm 659,7$ & $4326 \pm 353,9$ \\
24 horas & $4794 \pm 510,9^{\mathrm{a}}$ & $4388 \pm 656,2$ & $4116 \pm 332,9^{\mathrm{b}}$ \\
48 horas & $4969 \pm 554,8^{\mathrm{c}}$ & $4606 \pm 623,6$ & $4356 \pm 375,5^{\mathrm{d}}$ \\
72 horas & $5244 \pm 692,0$ & $4906 \pm 698,2$ & $4643 \pm 465,6$ \\
\hline
\end{tabular}

(Medias \pm DE. Letras diferentes en la misma línea indican diferencias significativas entre los grupos).

Cuadro 5. Glucemia de los corderos (mg/dl) de los diferentes grupos.

Lamb glicaemia $(\mathrm{mg} / \mathrm{dl})$ of different groups.

\begin{tabular}{lccc}
\hline & Grupo Ración (R) & Grupo Esquileo (E) & Grupo Campo Natural (CN) \\
\hline Parto & $83,0 \pm 10,1^{\mathrm{a}}$ & $74,34 \pm 20,3$ & $60,46 \pm 16,8^{\mathrm{b}}$ \\
24 horas & $121,0 \pm 8,2$ & $123,83 \pm 11,3^{\mathrm{c}}$ & $98,93 \pm 15,0^{\mathrm{d}}$ \\
\hline
\end{tabular}

(Medias \pm DE. Letras diferentes en la misma línea indican diferencias significativas entre los grupos. Diferencias estadísticas: ${ }^{\mathrm{a}-\mathrm{b}} \mathrm{P}<0,007$ y ${ }^{\mathrm{c}-\mathrm{d}} \mathrm{P}<0,001$ ).

Cuadro 6. Peso (g) y energía (cal/g MS) de la grasa parda de los diferentes grupos. Brown fat weight ( $\mathrm{g}$ ) and energy (cal/g DM) of different groups.

\begin{tabular}{lccc}
\hline & Grupo Ración (R) & Grupo Esquileo (E) & Grupo Campo Natural (CN) \\
\hline Peso & $28,63 \pm 7,59$ & $26,12 \pm 2,68$ & $25,39 \pm 3,88$ \\
Energía & $7505,4 \pm 627,1$ & $7968,7 \pm 347,7$ & $7359,0 \pm 462,9$ \\
\hline
\end{tabular}

(Medias \pm DE. Letras diferentes en la misma línea indican diferencias significativas entre los grupos).

en el grupo R, de 250 a $670 \mathrm{ml}$ para el grupo E y de 150 a $600 \mathrm{ml}$ para las ovejas alimentadas con pasto natural.

La supervivencia de los corderos fue del $100 \%$, ya que a las 72 horas postparto no había muerto ningún cordero en ninguno de los grupos experimentales.

Los pesos de los corderos se muestran en el cuadro 4. En todos los momentos en que se registraron los pesos los corderos nacidos de las ovejas suplementadas con ración (R) fueron en promedio los más pesados, mientras que los nacidos de las ovejas del grupo $\mathrm{CN}$ fueron en promedio los más livianos. Estas diferencias, a las 24 y 48 horas, tuvieron significación estadística (cuadro 4). Al calcular la ganancia relativa de peso se observa que los corderos nacidos del grupo $\mathrm{R}$ lograron una ganancia de peso a las 72 horas de vida de un $15,31 \%$ con respecto al peso al nacimiento, mientras que para los nacidos de los grupos $\mathrm{E}$ y CN esta ganancia fue respectivamente de $9,93 \%$ y $6,83 \%$.
Los corderos nacidos de los grupos $\mathrm{R}$ y E presentaron valores de glucemia, en el momento del parto, superiores a los nacidos del grupo $\mathrm{CN}$, esta diferencia tuvo carácter significativo $(\mathrm{P}<0,007)$ únicamente entre los grupos $\mathrm{R} y$ CN. La misma tendencia fue comprobada en la glucemia de los corderos medida a las 24 horas de vida. En ese momento hemos encontrado diferencias significativas $(\mathrm{P}<0,001)$ entre los grupos E y CN (cuadro 5).

El peso y la energía de la grasa parda perirrenal de los corderos eutanasiados se muestran en el cuadro 6. Los corderos nacidos de ovejas esquiladas (E) presentaron más calorías por gramo de materia seca en las muestras de grasa que los corderos de los grupos $\mathrm{R}$ y $\mathrm{CN}$, sin que estas diferencias fueran estadísticamente significativas. Los corderos del grupo $\mathrm{R}$ presentaron más energía bruta en la grasa parda que la de los del grupo $\mathrm{CN}$, pero esta diferencia tampoco logró tener significación estadística. 


\section{DISCUSIÓN}

Según el análisis de la alimentación suministrada a las ovejas, las madres alimentadas exclusivamente con pradera natural $(\mathrm{CN})$ fueron las que recibieron menor aporte de energía y de proteínas durante el último tercio de la gestación. Es posible que este aporte de nutrientes no lograse cubrir totalmente los requerimientos energéticos de la fase final de la preñez, lo que explica los altos valores de BHOB y el menor peso registrado en el día 140 de gestación. Tanto la alimentación suministrada a las ovejas del grupo R como la esquila realizada a las ovejas del grupo E permitieron un aumento de peso similar al propuesto por Bonino y col (1987), quienes sugieren que las ovejas al final del período de gestación deberían aumentar un kilo por semana y sobre todo evitar perder peso. Resultados similares fueron reportados por Chandler y col (1985) quienes encontraron que la media de peso en ovejas gestando un solo feto se incrementó alrededor de tres kilos durante los últimos 20 días de preñez. Sin embargo, las ovejas del grupo $\mathrm{CN}$ no mostraron aumento del peso corporal durante el último tercio de la gestación, según Borrelli (2001), debido a que si la demanda energética es superior a la energía ingerida la oveja moviliza y consume sus reservas grasas, lo que conduce a una pérdida de peso.

Como consecuencia del importante crecimiento fetal en las últimas semanas de gestación la oveja experimenta un aumento de sus necesidades nutritivas, y especialmente de energía, lo que obliga a ésta a aumentar el consumo de nutrientes, a incrementar la eficiencia de utilización de esos nutrientes por los tejidos e incluso a movilizar, si es preciso, sus reservas corporales (Gibbons 1996).

En opinión de Russell (1984), las concentraciones de BHOB son un buen indicador de la nutrición en ovinos criados en condiciones extensivas. Teniendo en cuenta las clasificaciones propuestas por Russell y col (1977) y por Andrews (1997) observamos que las ovejas pertenecientes al grupo $\mathrm{CN}(\mathrm{BHOB}=0,91 \pm 0,41 \mathrm{mmol} / \mathrm{l})$ presentaron al parto una condición de subnutrición moderada, en tanto las ovejas del grupo $\mathrm{R}(\mathrm{BHOB}=0,47 \pm 0,21 \mathrm{mmol} / \mathrm{l})$ y las del grupo $\mathrm{E}(\mathrm{BHOB}=0,69 \pm 0,25 \mathrm{mmol} / \mathrm{l})$ presentaron valores de nutrición normal.

La diferencia encontrada en este cuerpo cetónico, en el momento del parto, entre las ovejas de los grupos R y E se explica porque en las últimas semanas de gestación la capacidad física del rumen se ve comprometida como consecuencia del incremento de tamaño del útero gestante (Gibbons 1996, Andrews 1997, Rook 2000). Si bien la esquila preparto y el estrés por frío que es capaz de provocar elevan el consumo de alimento de estos animales (Vipond y col 1987) la menor capacidad ruminal disminuye la capacidad de ingesta, a lo que se suma la mala calidad del pasto. Esto no sucedió en las ovejas del grupo R, ya que su alimentación se suplementó con un concentrado energético. Symonds y col (1986) además concluyen que las ovejas preñadas esquiladas antes del parto incrementan de forma manifiesta sus requerimientos energéticos, que satisfacen en gran medida mediante la oxidación de los depósitos de grasa corporal.

El aumento de las demandas fetales de nutrientes, y especialmente de glucosa, en las últimas semanas de gestación explicaría la disminución de los valores de glucemia en las ovejas de los tres grupos en el día 140 de gestación (Gibbons 1996, Montossi y col 2005). La glucemia fue más elevada en las ovejas del grupo suplementado con concentrado (R), mientras que las alimentadas exclusivamente con pasto natural $(\mathrm{CN})$ mostraron la glucemia más baja, lo que concuerda con los resultados de Symonds y col (1986) en ovejas con esquila preparto, y con Banchero y col $\left(2004^{\mathrm{a}}\right)$ al suplementar la alimentación de las ovejas al final de la gestación. El aporte extra de suplementos energéticos provoca un incremento considerable de ácidos grasos volátiles, principalmente propiónico, que incrementa los niveles de glucosa en sangre vía neoglucogénesis (Ndi bualonji y Godeau 1993), y especialmente porque la intensidad de la neoglucogénesis depende en primer lugar del nivel de alimentación (Baird y col 1983). Las ovejas esquiladas antes del parto mostraron una tendencia a aumentar su glucemia, posiblemente debido a que la exposición al frío inhibe la secreción de insulina, dando lugar a un aumento de la glucosa en sangre (Symonds y col 1986, Symonds y col 1988). Además la exposición continuada al frío produce una disminución de la relación insulina:glucagón plasmático, lo que estimula la neoglucogénesis hepática (Symonds y col 1988).

Aunque sin mostrar diferencias significativas el volumen de calostro recogido tanto en el día del parto, como 24 horas más tarde, fue mayor en las ovejas del grupo suplementado (R) que en las ovejas que únicamente ingerían pasto. Así, en todos los grupos, la cantidad de calostro producido 24 horas tras el parto incluso duplica la cantidad recogida inmediatamente tras el parto. Por tanto, se evidencia una respuesta positiva, en la producción de calostro, por las madres a la suplementación y que puede explicarse al tener en cuenta la glucemia de las ovejas (Pattinson 1991, Banchero y col 2004a , Banchero 2005 y Banchero y col 2006). En opinión de Banchero y col (2004 ${ }^{\mathrm{a}}$ y y Banchero (2005) el volumen de calostro producido se relaciona con la disponibilidad de glucosa para la síntesis de lactosa, la cual al ser un azúcar osmóticamente activo incrementa el paso de líquido hacia la glándula mamaria, y por tanto produciendo una mayor cantidad y una consistencia mucho más líquida del calostro, facilitando así al amamantamiento del cordero.

Existe una relación positiva entre el consumo de energía y el flujo sanguíneo hacia el hígado, lo que da lugar a un mayor catabolismo de hormonas antagónicas a la producción de lactosa, como puede ser la progesterona. Esta hormona inhibe la síntesis de lactosa y, por ende, su disminución provoca un mayor volumen de calostro acumulado (Banchero y col 2004 ${ }^{\mathrm{a}}$ ). La cantidad de calostro obtenido de las ovejas en ambos ordeños mostró una 
importante variación entre los individuos, e incluso entre ovejas pertenecientes al mismo grupo. Esta particularidad también fue observada por O'Doherty y col (1997), por Chirstley y col 2003 y por Pattinson y Thomas (2004), quienes lo atribuyeron a diferencias genéticas individuales.

El peso vivo al nacimiento de los corderos de los tres grupos experimentales se ubicó dentro del considerado como óptimo por varios autores (Dalton y col 1980, Corner y col 2005, Montossi y col 2005, Corner y col 2007). El rango de peso al parto, que teóricamente aumentaría la supervivencia de los corderos, se situó entre 3,5 y 5,5 kg. Aquellos corderos nacidos con peso inferior poseerían menores reservas energéticas para contrarrestar las pérdidas de temperatura y menor vigor. Como consecuencia se incrementaría el tiempo preciso para incorporarse y para amamantarse (Gibbons 1996). Los corderos que al parto superaran este peso tenderían a provocar partos distócicos (Montossi y col 2005).

Las diferencias en el peso al nacimiento de los corderos de los distintos grupos han sido descritas por varios investigadores (Cardelino 1974, Chirstley y col 2003, Encinias y col 2004, Banchero y col 2004 ${ }^{\mathrm{a}}$, McMullen y col 2005, Banchero y col 2006). Sin embargo no existe consenso sobre el efecto de la suplementación y del esquileo sobre los corderos. Según Revell y col (2000) la esquila preparto temprana incrementa significativamente el peso de los corderos mellizos y no afecta al peso vivo de corderos de partos simples. Sin embargo también se ha señalado un incremento significativo del peso vivo al nacimiento en corderos únicos hijos de madres suplementadas, así como de aquellas esquiladas antes del parto (Cardelino 1974, Robinson 1983, Symonds y col 1986, Revell y col 2000, Osgerby y col 2002, Banchero y col $2004^{\mathrm{b}}$, Rhind 2004, Corner y col 2005, Corner y col 2007). Posiblemente el mayor peso al nacimiento en corderos nacidos de madres suplementadas se debería a la mayor disponibilidad de energía y de proteínas, que son destinadas al crecimiento fetal, si un balance positivo durante las fases finales de la gestación (Russel 1984, Gibbons 1996, Montossi y col 2005).

El esquileo puede provocar un mayor ingreso de glucosa no insulinodependiente a la unidad placentofetal (Revell y col 2000), a lo que se suma que el estrés por frío inducido por la esquila inhibe la secreción de insulina, lo que provoca un aumento de la glucemia materna (Symonds y col 1986, Revell y col 2000). Montossi y col (2005) sugieren que la placenta tiene un papel esencial en el control de los nutrientes al feto, lo que condiciona el peso al nacer del cordero y que podría justificar el mayor peso de los corderos procedentes de ovejas esquiladas. El tamaño de la placenta y el número y tamaño de los cotiledones pueden verse afectados por el esquileo preparto y por el manejo nutricional durante la preñez. Además la placenta crece a partir del día 30 hasta llegar al desarrollo máximo aproximadamente en el día 90 de gestación, momento en el cual su tamaño se estabiliza. Así si la esquila se realiza entre los días 60 y 90 de la gestación, la respuesta en los pesos de los corderos al nacimiento será mayor (Montossi y col 2005). Osgerby y col (2002) proponen que la disminución de la masa placentaria, la concentración de glucosa amniótica y alantoidea y los niveles maternos de glucosa plasmática en ovejas subalimentadas en la última etapa de la gestación pueden comprometer el desarrollo fetal. De forma similar, el efecto de la subnutrición en ovejas gestantes sobre el peso de sus corderos al parto depende de la severidad y de su duración, así como de la condición corporal de las ovejas en el momento que comenzó (McMullen y col 2005). La subnutrición tiene un efecto más marcado sobre el peso vivo de la oveja que sobre el peso fetal, demostrando la habilidad de la madre para mantener los aportes nutricionales al feto, incluso a expensas de sus reservas corporales (Gibbons 1996). La ganancia relativa de peso en las primeras 72 horas de vida de los corderos de los diferentes grupos puede deberse a las reservas energéticas presentes al nacimiento, al consumo de esas reservas para soportar las condiciones medioambientales durante ese período y al aporte energético del calostro.

Los valores más elevados de glucemia al parto en los corderos nacidos de las ovejas de los grupos $\mathrm{R}$ y $\mathrm{E}$ se relacionan con una mayor concentración de glucosa sérica disponible, en los últimos días de la gestación, en las ovejas de estos grupos con respecto a las de ovejas alimentadas exclusivamente con pasto $(\mathrm{CN})$. La glucosa sanguínea de los corderos a las 24 horas tras el parto mostró un comportamiento similar, lo cual puede ser atribuido a las mayores reservas energéticas de los corderos, y que se hace más visible en el grupo E. A partir de la segunda mitad de la gestación el exceso de carbohidratos es acumulado en el hígado y en los músculos de los corderos en forma de glucógeno, siendo la fuente energética de utilización inmediata durante las primeras horas de vida (Gibbons 1996).

El peso de la grasa parda perirrenal de los corderos no mostró diferencias significativas entre los tres grupos. Resultados similares fueron obtenidos al comparar los pesos de la grasa parda perirrenal entre corderos nacidos de madres esquiladas en el preparto y ovejas sin esquilar (Clarke y col $1997^{\mathrm{b}}$ ) y entre corderos nacidos de madres suplementadas y sin suplementar (Encinias y col 2004). Sin embargo, Symonds y Clarke (1998) demostraron que los corderos nacidos de ovejas livianas tenían menos cantidad de tejido adiposo pardo que aquellos nacidos de ovejas pesadas. Estos corderos pudieron adaptar su temperatura corporal cuando nacían a temperatura ambiente benigna, pero no lograron adaptarse en ambiente frío, padeciendo hipotermia. Observamos que los datos obtenidos de la energía de la grasa parda perirrenal, si bien son tendencias, son coincidentes con los descritos por Clarke y col (1997b). Estos investigadores indican que la grasa parda perirrenal de los corderos nacidos de madres esquiladas mostró una superior actividad termogénica del tejido adiposo pardo, 
además de exhibir una mayor concentración plasmática de triiodotironina al compararlos con corderos nacidos de madres no esquiladas. Para ello estimaron la actividad termogénica de la grasa parda mediante el análisis de contenido de UCP-1 y de las catecolaminas presentes (Clarke y col $1997^{b}$ ) mientras que nosotros utilizamos una bomba calorimétrica.

Los resultados de este trabajo sugieren que tanto la suplementación energética de las ovejas en el último tercio de gestación como el esquileo preparto (en el día 110 de preñez) repercuten positivamente en el metabolismo energético de las ovejas al parto. Los corderos nacidos de madres suplementadas nacen con mayor peso corporal y mayor glucemia y los nacidos de madres esquiladas poseen grasa parda con mayor eficiencia energética. Ambas situaciones permitirían a los corderos afrontar en mejores condiciones las posibles adversidades medioambientales en sus primeras 72 horas de vida.

\section{RESUMEN}

El objetivo fue evaluar los efectos de la alimentación y el esquileo, durante el último tercio de la gestación, sobre el metabolismo energético de las ovejas y su repercusión sobre las reservas energéticas de sus corderos. A los 90 días de gestación 38 ovejas Corriedale adultas, gestando un solo feto, fueron divididas en dos grupos, $\mathrm{R}(\mathrm{n}=12$, suplementado con concentrado) y $\mathrm{CN}(\mathrm{n}=26$, alimentado a campo natural), evaluándose su estado metabólico mediante determinaciones de la glucemia y del ß-hidroxibutirato. A los 110 días de gestación se esquiló a la mitad de las ovejas del grupo $\mathrm{CN}$, quedando dividido en dos nuevos grupos (E, esquiladas, $\mathrm{n}=13$ y campo natural, $\mathrm{CN}, \mathrm{n}=13$ ), estudiándose el efecto de la esquileo sobre los mismos parámetros. En los corderos se determinó la glucemia (al parto y a las 24 horas), el peso (al parto, a las 24,48 y 72 horas) y el peso y valor energético de grasa parda perirrenal. En el día 140 de gestación las ovejas alimentadas con concentrado y las esquiladas presentaron valores superiores de glucemia que las alimentadas exclusivamente con pasto natural; los valores de $\beta$-hidroxibutirato sérico fueron más elevados en las ovejas de pasto natural y las esquiladas que las suplementadas con concentrado. Al nacimiento el peso de los corderos no presentó diferencias entre los tres grupos. La glucemia de los corderos nacidos de ovejas suplementadas con concentrado fue mayor que los de ovejas de pasto natural, al parto y a las 24 horas y en las esquiladas mayor en que se alimentaban exclusivamente de pasto a las 24 horas. Los corderos de las ovejas esquiladas presentaron grasa parda con mayor valor energético que los provenientes de ovejas que pastaban exclusivamente hierba, demostrándose así relación entre el estado energético de las ovejas al parto y las reservas energéticas con las que nacen sus corderos.

\section{AGRADECIMIENTOS}

Este trabajo fue posible gracias a la colaboración del Dr. Bruno López, Director del Campo Experimental $N^{\circ} 2$ de la Facultad de Veterinaria y al personal de dicha institución, especialmente el Sr. Gustavo Cazard. También deseamos agradecer la colaboración del personal del Laboratorio de Análisis Clínicos y de Nutrición de la Facultad de Veterinaria. El valor energético de la grasa parda de los corderos se procesó en una bomba calorimétrica en el Laboratorio de Nutrición de la Facultad de Agronomía gracias a la desinteresada colaboración de la Dra. Cristina Cabrera. Agradecemos asimismo a la Comisión Sectorial de Investigación Científica (CSIC), Universidad de la República, por su apoyo en la financiación de la experiencia.

\section{REFERENCIAS}

Andrews A. 1997. Pregnancy toxaemia in the ewe. In practice 19, 306-312.

Avram AS, MM Avram, JD James, W James. 2005. Subcutaneous fat in normal and diseased states. Anatomy and physiology of white and brown adipose tissue. J Amer Acad Dermat 53, 671-683.

Azzarini M. 1974. Mortalidad de corderos. En: Relevamiento básico de la producción ovina del Uruguay en 1972-1973. SUL, Montevideo, Uruguay, Pp 15-19.

Baird GD, JO Van der Walt, En Bergman. 1983. Whole-body metabolism of glucosa and lactate in productive sheeps and cows. $\mathrm{Br} \mathrm{J}$ Nutr 50, 249-265.

Banchero G, R Perez, R Bencini, D Lindsay, J Milton, G Martin. 2006. Endocrine and metabolic factors involved in the effect of nutrition on the production of colostrum in female sheep. Reprod Nutr Dev 46, 447-460.

Banchero G. 2005. Alimentación estratégica para mejorar la lactogénesis y el comportamiento de la oveja al parto. Resúmenes de las XXXIII Jornadas Uruguayas de Buiatría, Paysandú, Uruguay, Pp 72-78.

Banchero GE, G Quintans, GB Martin, DR Lindsay, JTB Milton. 2004. Nutrition and colostrum production in sheep. 1. Metabolic and hormonal responses to high energy supplement in the final stages of pregnancy. Reprod Fert and Dev 16, 633-643.

Banchero GE, G Quintans, GB Martin, JTB Milton, DR Lindsay. 2004. Nutrition and colostrum production in sheep. 2. Metabolic and hormonal responses to different energy sources in the final stages of pregnancy. Reprod Fert and Dev 16, 645-653.

Bell AW. 1995. Regulation of organic nutrient metabolism during transition from late pregnancy to early lactation. J Anim Sci 73, 2804-2819.

Bonino J, R Sienra, L Sorondo. 1987. Enfermedades causadas por trastornos metabólicos: toxemia de la preñez. En: Bonino J, Durán del Campo A, Mari JJ (eds). Enfermedades de los lanares. Hemisferio Sur, Tomo II, Pp 239-265.

Borrelli P. 2001. Producción animal sobre pastizales naturales. En: Borrelli P, Oliva G (eds). Ganadería sustentable en la Patagonia Austral. INTA, Reg. Pat. Sur, Pp 126-160.

Buckrell BC. 1988. Application of ultrasonography in reproduction in sheep and goats. Theriogenology 29, 11-20.

Cardelino R. 1974. Relevamiento de la producción ovina del Uruguay. En: Relevamiento básico de la producción ovina del Uruguay en 1972-1973. SUL, Montevideo, Uruguay, Pp 7-13.

Chandler KD, BJ Leury, AR Bird, AW Bell. 1985. Effects of udernutrition and exercise during late pregnancy on uterine, fetal and uteroplacental metabolism in the ewe. Bri J Nutr 53, 625-635.

Chirstley R, KL Morgan, TD Parkin, NP French. 2003. Factors related to the risk of neonatal mortality, birth weight and serum immunoglobulin concentration in lambs in the UK. Prev Vet Med, 57, 209-226.

Clarke L, DP Yakubu, ME Symonds. $1997^{\mathrm{c}}$. Influence of maternal bodyweight on size, conformation and survival of newborn lambs. Reprod Fert Dev 5, 509-514.

Clarke L, DS Buss, DT Juniper, MA Lomax, ME Symonds. 1997 Adipose tissue development during early postnatal life in ewe-reared lambs. Exp Physio 6, 1015-1027.

Clarke L, ME Symonds. 1998. Thermoregulation in newborn lambs: influence of feeding and ambient temperature on brown adipose tissue. Exp Physiol 83, 651-657.

Clarke L, MJ Briant, MA Lomax, ME Symonds. 1997ª. Maternal manipulation of brown adipose tissue and liver development in the ovine fetus during late gestation. Br J Nutr 6, 871-883.

Corner RA, PR Kenyon, KJ Stafford, DM West, MH Oliver. 2005. The effect of mid-pregnancy shearing or yarding stress on ewe post-natal behaviour and the birth weight and post-natal behaviour of their lambs. Livest Sci 102, 121-129.

Corner RA, PR Kenyon, KJ Stafford, DM West, MH Oliver. 2007. The effect of mid-pregnancy shearing and litter size on lamb birth 
weight and postnatal plasma cortisol response. Small Ruminant Res 73, 115-121.

Dalton DC, TW Knight, DL Johnson. 1980. Lamb survival in sheep breeds on New Zealand hill country. New Zealand J Agric Res 32, 167-173.

Dennis SM. 1974. Perinatal lamb mortality in Western Australia. I. General procedures and results. Aust Vet J 50, 443-449.

Dwyer CM, CA Morgan. 2006. Maintenance of body temperature in the neonatal lamb: Effects of breed, birth weight, and litter size. $J$ Anim Sci 84, 1093-1101.

Encinias H, G Lardy, A Encinias, M Bauer. 2004. High linoleic acid safflower seed supplementation for gestating ewes: Effects on ewe performance, lamb survival, and brown fat stores. J Anim Sci 82, 3654-3661.

Faurie A, D Mitchell, H Laburn. 2004. Peripartum body temperatures in free-ranging ewes (Ovis aries) and their lambs. J Therm Biol $29,115-122$.

Gate JJ, L Clarke, MA Lomax, ME Symonds. 1999. Chronic cold exposure has no effect on brown adipose tissue in newborn lambs born to well-fed ewes. Reprod Fert Dev 8, 415-418.

Gibbons A. 1996. Efecto de la esquila sobre el peso al nacimiento de los corderos Merino en el sistema extensivo patagónico. Trabajo Monográfico, Curso superior de producción animal, producción y alimentación, Instituto Agronómico Mediterráneo de Zaragoza, Zaragoza, España.

Lyndsay DB, BP Setchell. 1976. The oxydation of glucose, ketone bodies and acetate by the brain of normal and ketonaemic sheep. J Physiol 3, 801-823.

Mari JJ. 1987. Enfermedades que afectan la supervivencia del cordero. En: Bonino J, A Durán del Campo, JJ Mari (eds). Enfermedades de los lanares. Tomo III. Hemisferio Sur, Montevideo, Uruguay, Pp 73-99.

McFarlane D. 1965. Perinatal lamb losses. I An autopsy method for the investigation of perinatal losses. New Zealand Vet J 13, 116-120.

McMullen S, J Osgerby, J Milne, J Wallace, D Wathes. 2005. The effects of acute nutrient restriction in the mid-gestational ewe on maternal and fetal nutrient status, the expression of placental growth factors and fetal growth. Placenta 26, 25-33.

Montossi F, I de Barbieri, A Digiero, H Martínez, M Nolla, S Luzardo, A Mederos, R San Julián, W Saint, J Levratto, J Furgón, G Lima, J Costales. 2005. La esquila preparto temprana: Una nueva opción para la mejora reproductiva ovina. En: Seminario de actualización técnica. Reproducción ovina: recientes avances realizados por el INIA. Treinta y Tres, Uruguay, Pp 85-102.
Ndi bualonji BB, JM Godeau. 1993. La neoglucogenese et les acides amines chez les ruminants: revue. Ann Med Vet, 137, 537-554.

O'Doherty JV, TF Crosby. 1997. The effect of diet in late pregnancy on colostrum production and immunoglobulin absortion in sheep. Anim Sci 64, 87-96.

Osgerby JC, DC Wathes, D Howard, TS Gadd. 2002. The effect of maternal undernutrition on ovine fetal growth. $J$ Endocrinol 1, 131-141.

Pattinson SE, EW Thomas. 2004. The effect of sire breed on colostrum production of crossbred ewes. Livestock Prod Sci 86, 47-53.

Pattinson S, DAR Davies, AC Winter. 1991. Calostrum production by prolific ewes. Animal Prod, 52, 583.

Pell JM, EN Bergman. 1983. Cerebral metabolism of amino acids and glucose in fed and fasted sheep. Amer J Physiol 224, 282-289.

Revell DK, SF Main, BH Breier, YH Cottam, M Hennies, SN McCutcheon. 2000. Metabolic responses to mid-pregnancy shearing that are associated with a selective increase in the birth weight of twin lambs. Domest Anim Endocrinol 18, 409-422.

Rhind SH. 2004. Effects of maternal nutrition on fetal and neonatal reproductive development and function. Anim Reprod Sci 82, 169-181.

Robinson JJ. 1983. Nutrition of the pregnant ewe. In: Haresign W (ed). Sheep Production. London, Butterworths, Pp 111-131.

Robinson JJ. 1996. Nutrition and reproduction. Am Reprod Sci 42, 25-34.

Rook JS. 2000. Pregnancy toxemia of ewes, does, and beef cows. Vet Clin N Am: Food Animal Practice 16, 293-317.

Russell AFJ, JM Doney, RL Reid. 1977. The use of biochemical parameters in controlling nutrition state in pregnancy ewes and the effect of undernourishment during pregnancy of lamb birth weight. J Agric Camb 68, 351-358.

Russell AJF. 1984. Means of assessing the adequacy of nutrition of pregnant ewes. Livestock Prod Sci 11, 429-436.

Stephenson T, H Budge, A Mostyn, S Pearce, R Webb, ME Symonds. 2001. Fetal and neonatal adipose maturation: A primary site of cytokine and cytokine-receptor action. Biochem Soc Trans 29, 80-85.

Symonds E, J Bryant, D Shepherd, M Lomax. 1988. Glucose metabolism in shorn and unshorn pregnant sheep. Br J Nutr 60, 249-263.

Symonds ME, L Clarke. 1998. Influence of maternal bodyweigth on adaptation after birth in near-term lambs delivered by Caesarean section. Reprod Fertil Dev 10, 333-339.

Symonds ME, MJ Bryant, MA Lomax. 1986. The effect of shearing on the energy metabolism of the pregnant ewe. Br J Nutr 56, 635-643.

Vipond JE, ME King, DM Inglis. 1987. The effect of winter shearing of housed pregnant ewes on food intake and animal performance. Anim Prod 45, 211-221. 